\title{
Effects of IRW and IQW on Oxidative Stress and Gut Microbiota in Dextran Sodium Sulfate-Induced Colitis
}

\author{
Gang Liua ${ }^{a}$ Wenxin Yan ${ }^{a}$ Sujuan Ding ${ }^{a}$ Hongmei Jiang ${ }^{a} \quad$ Yong Ma ${ }^{a, c}$ \\ Hua Wang ${ }^{a}$ Jun Fanga \\ ${ }^{a}$ College of bioscience and biotechnology, Hunan Agricultural University, Changsha, ${ }^{b H u n a n ~ P r o v i n c i a l ~}$ \\ Key Laboratory of Animal Nutritional Physiology and Metabolic Process, Key Laboratory of Agro- \\ ecological Processes in Subtropical Region, Institute of Subtropical Agriculture, Chinese Academy of \\ Sciences, National Engineering Laboratory for Pollution Control and Waste Utilization in Livestock \\ and Poultry Production, Changsha, 'Academician Workstation of Hunan Baodong Farming Co. Ltd., \\ Changsha, China
}

\section{Key Words}

Inflammatory bowel disease (IBD) $\bullet$ Microbiota $\bullet$ IRW $\bullet$ IQW

\begin{abstract}
Background/Aims: There are known links between inflammatory bowel disease (IBD) and changes in the microbiota of the gut and inflammation and oxidative stress. In this study, a colitis model induced by dextran sodium sulfate (DSS) in mice is used to evaluate whether the presence of bioactive peptides IRW (Ile-Arg-Trp) and IQW (Ile-Gln-Trp) peptides is advantageous. Methods: The mice were arbitrarily assigned to the following four groups: (i) control (untreated), (ii) dextran sodium sulfate (DSS) treated, (iii) IRW-DSS treated, and (iv) IQW-DSS treated. For 7 days, the control group subjects had unrestricted access to untreated drinking water, whereas the drinking water supplied to the subjects in the DSS, IRW-DSS, and IQW-DSS groups during this period consisted of 5\% DSS solution. The colonic lesions were scored after hematoxylin and eosin staining. Serum antioxidant capacity was analyzed by 2,2'-azino-bis(3-ethylbenz-thiazoline-6-sulfonic acid) (ABTS) radical cation decolorization test and the microbiota in the colonic contents were sequenced by HiSeq2500 PE250. Results: The presence of DSS reduced daily weight gain, enhanced histopathology scores, and inhibited antioxidant enzyme expression. Superoxide dismutase, catalase, and glutathione peroxidase activities in the DSS-induced colitis model were significantly enhanced $(P<0.05)$ in the presence of dietary IRW and IQW. Furthermore, the Simpson index was significantly increased $(P<0.05)$ in the presence of dietary IRW and IQW compared to the control group. IRW and IQW increased the abundance of Coprococcus_1, Ruminococcaceae_UCG-014, and Desulfovibrio compared to the control group and DSS group. Furthermore, IQW decreased the abundance of Bacteroides in relation to the control group, but increased Parabacteroides.

G. Liu and W. Yan contributed equally to this work.

Sujuan Ding, Hongmei Jiang and Jun Fang
\end{abstract}

\section{KARGER}


In addition, IRW increased the level of Anaerotruncus, Oscillibacter, and Ruminiclostridium_9 compared to the control group. Conclusion: This study concludes that the presence of IRW or IQW can mitigate DSS-induced oxidative stress by improving the activities of antioxidant enzymes, increasing intestinal microbial diversity and enhancing the abundance of gut microbiota, which may help maintain the homeostasis of host health and microenvironment in a DSS-induced mouse model, thus providing a potential further treatment for IBD patients.

(C) 2018 The Author(s)

Published by S. Karger AG, Basel

\section{Introduction}

Inflammatory bowel diseases (IBD) comprise a variety of persistent and recurrent ailments of the gastrointestinal tract leading to major inflammation and injury to the rectum and gut. Two significant forms of IBD are Crohn's disease (CD), recognized by chronic intermittent transmutal inflammation usually involving the terminal ileum but potentially affecting any part of the gastrointestinal tract, and ulcerative colitis (UC), recognized by inflammation of the colonic mucosa that varies in full extent from the rectum to the proximal colon [1]. Given that the determinants of genetic susceptibility are moderately consistent within the community at large, the pathogenesis and rising incidence of CD and UC worldwide are probably strongly dependent upon environmental determinants. The links between IBD and a range of environmental determinants, such as diet and the use of antibiotics [2], social standing and early life exposure to microorganisms [3], and exposure to microorganisms throughout life, are evidenced by epidemiological, clinical, and experimental research. Several of these determinants may have a significant impact on the range of microbiota present in the gut [4].

Significant differences between the microbiota of IBD patients and healthy individuals, such as reduced diversity and bacterial load along with the development of various characteristics of the condition known as dysbiosis, have been demonstrated via the examination of the microbiome using 16S rRNA sequencing [4-6]. Further indepth investigations on $C D$ and UC sufferers have demonstrated measurable decreases in Firmicutes (particularly Clostridium groups) and numerous beneficial bacterial species from genera such as Bacteriodes, Eubacterium, and Lactobacillus and an increase in Proteobacteria [6]. Studies have also shown that the matrix metalloproteinases, which are the primary mediators of tissue damage and are responsible for provoking matrix degradation, epithelial cell detachment, and ulceration, are activated by the inflammation response [7]. Although evidence suggests that microbes are essential in driving the inflammatory process, some species from a range of genera, such as Bifidobacterium, Faecalibacterium, and Lactobacillus, may in fact safeguard their hosts' mucosa from unwarranted and potentially harmful inflammatory responses [8-10].

Although a number of medical treatments, such as steroids, immunomodulators, and antibodies, are available to IBD patients, their application is restricted by safety issues surrounding detrimental effects, such as malignancy and infection [11, 12]. Studies have demonstrated the successful inhibition of cytokine-induced inflammatory protein expression in vascular endothelium, at least in part via the modulation of the NF- $\kappa$ B pathway, in the presence of two novel peptides, namely IRW and IQW, which are derived from the egg white protein ovotransferrin [13-15]. In addition to this anti-inflammatory effect, the observed advantages of these peptides have included antioxidant effects [16]. The capacity for dietary constituents to function as modulators for intestinal inflammatory disease has been demonstrated by previous work examining nutritional regulation by amino acids [1719]. This study examines the impacts of the presence of dietary IRW or IQW on oxidative stress and colon microbiota in dextran sodium sulfate (DSS) induced colitis. 


\section{Cellular Physiology Cell Physiol Biochem 2018;51:441-451

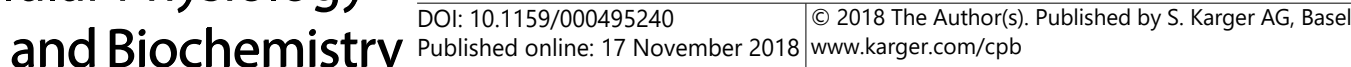

Liu et al.: Effects of IRW and IQW on IBD

\section{Materials and Methods}

\section{Experimental design}

The Chinese guidelines for animal welfare were observed in the design of the experimental strategy. The approval of the Animal Care and Use Committee of Hunan Agricultural University was obtained. The experiment used 40 female ICR mice weighing approximately $22.5 \mathrm{~g}$. The subjects were arbitrarily assigned to the following four groups: (i) the control group ( $n=10)$, (ii) the DSS-treated group ( $n=10)$, (iii) the IRW-DSS group ( $\mathrm{n}=10)$, and (iv) the IQW-DSS group $(\mathrm{n}=10)$. All of the mice were kept in polycarbonate cages in a room with a 12 -h cycle of light and dark under conditions of controlled temperature $\left(25 \pm 3{ }^{\circ} \mathrm{C}\right)$ and humidity $(50 \pm 5 \%)$. The mice in groups (i) and (ii) were given a basic diet and ready access to clean drinking water for 7 days, whereas the mice in groups (iii) and (iv) received a basic diet with drinking water containing $30 \mathrm{mg} / \mathrm{ml}$ IRW or IWQ for the same period of time. Commencing on day 7, all of the mice, except for those in group (i), were given ready access to drinking water containing 5\% DSS solution for a further 7 days [17]. This drinking water was prepared by dissolving DSS (molecular weight 5, 000, supplied by Kayon Biological Technology Co. Ltd.) in tap water to obtain a 5\% (wt/vol) DSS solution. On the morning of day 15 (the end of the experimental period), each mouse was weighed to assess the average daily weight gain and then each mouse was sacrificed and colon length and weight were measured as detailed in our previous publication [17]. Colon tissues and contents from each mouse were also collected, immediately frozen in liquid nitrogen and retained at $-70^{\circ} \mathrm{C}$ for further analysis.

\section{Histological analysis of colon tissues}

The terminal colon was removed and fixed in $10 \%$ formalin, after which paraffin-embedded sections were prepared and stained with hematoxylin and eosin at Hunan Agricultural University for histological grading. Scores were assigned ranging from 0 (no change) to 4 (extensive infiltration and tissue damage) based on the examination of the following six criteria: cryptitis, erosion, edema, goblet cell hyperplasia, inflammation, and ulceration. Lesions were scored from 0 to 4 , where $0=$ no colitis or epithelial thickening; 1 = increased number of mucosal leukocytes and/or minor epithelial cell hyperplasia; 2 = multiple inflammation sites, leukocytic infiltration of mucosa and submucosa, and/or noticeable epithelial cell hyperplasia (increase in crypts by 2 to 3 times); 3 = major leukocytic infiltration of mucosa and submucosa, ulceration, depletion of mucin-secreting goblet cells, and/or significant epithelial cell hyperplasia (increase in crypts by 3 to 10 times); and 4 = extensive transmural leukocytic infiltration, crypt abscesses, and/or extreme epithelial cell hyperplasia (increase in crypts by more than 10 times).

\section{Serum antioxidant capacity and antioxidant enzymes}

The 2, 2'-azino-bis(3-ethylbenz-thiazoline-6-sulfonic acid) (ABTS) radical cation decolorization test was used to measure the total antioxidant capacity of mice treated as described in the previous sections. For this procedure, a mixture containing $10 \mathrm{mM}$ ABTS solution and $2 \mathrm{mM}$ hydrogen peroxide was prepared and stored overnight in the dark at $4{ }^{\circ} \mathrm{C}$. The resulting ABTS $\bullet+$ solution was then diluted by a ratio of 1:10 to acquire an absorbance of approximately 0.31 at $660 \mathrm{~nm}$. A buffer (1, $000 \mu \mathrm{L}, \mathrm{pH} 3.6)$, plasma $(10 \mu \mathrm{L})$, and ABTS $-(25 \mu \mathrm{L})$ were sequentially added to a cuvette and the absorbance at $660 \mathrm{~nm}$ was measured after 5 min. A blank was included in each assay and the tests were carried out in triplicate. The results are presented in micromolar equivalents of ascorbic acid, an antioxidant normally found in the plasma [20]. Superoxide dismutase (SOD), glutathione peroxidase (GPx), and catalase (CAT) kits supplied by the Jiancheng Bioengineering Institute, Nanjing, China, were used to evaluate the antioxidant enzyme activities, defined as the quantity of enzyme degrading $1 \mu \mathrm{mol}$ of substrate per minute [21]. The results are presented in units per mg of protein.

\section{Microbial community analysis}

The V3-V4 region of the 16S rRNA gene of the microorganism in the colonic contents was amplified after extracting DNA for pyrosequencing. Briefly, the V4-V5 region of the 16S rRNA gene of the intestinal microflora of piglets was amplified using specific primers 515F (5'-GTGCCAGCMGCCGCGGTAA-3') and 926R (5'-CCGTCAATTCMTTTGAGTTT-3'), which amplify almost all bacteria and archaea with few prejudices against specific groups. The PCR product was sequenced by HiSeq2500 PE250 (Illumina, U.S.). For this analysis, the raw data were screened and assembled using Quantitative Insights into Microbial Ecology 
(QIIME) (http://qiime.org/), an open-source software pipeline that uses PycGooTooKiT6 to handle the problem of sequential data from original sequences to interpretation and database deposition [22], and Fast Length Adjustment of SHort reads, a software tool that splices paired-end reads and extends the reads to find their correct overlap [23]. For each sample, the reads identified as chimeras were removed to generate a collection of effective sequences. The sequences were analyzed and the operational taxonomic units (OTUs) were determined with an identity threshold of 97\%, using the QIIME and UPARSE pipeline (http:// drive5.com/uparse) applications [22]. The RDP classifier (Version 2.2, http://sourceforge.net/projects/ rdpclassifier/) was used to assign taxonomic data to a selected representative sequence for each OTU [24]. The RDP classifier and the Greengenes database (http://greengenes.lbl.gov/cgi-bin/nph-index.cgi) were used to assign taxonomic classifications. The abundance-based coverage estimator (ACE), bias-corrected Chao1 richness estimator, Shannon and Simpson indices, and Good's coverage were used to obtain alpha diversity values for colon bacterial communities at a sequence depth of 31, 460 for all samples.

\section{Statistical analysis}

The Statistical Package for Social Science (SPSS v19.0, U.S.A.) was used to conduct the statistical analysis. One-way analysis of variance was used to evaluate the differences between groups, with the results presented as the mean \pm SEM. Differences were regarded as statistically significant when $P<0.05$. Before this analysis, a Shapiro-Wilk test was used to evaluate data normality, whereas the Levene test was used to verify the homogeneity of variance. A principal coordinate analysis of overall microbial diversity based on an un-weighted Unifrac metric was conducted using Bray-Curtis distance to compare all of the samples.

\section{Results}

The most frequent clinical index in IBD is weight loss. As such, the central strategy was to evaluate the average daily weight gain following DSS treatment. As indicated in Fig. 1A, the DSS group exhibited a significant reduction in daily weight gain $(P<0.05)$, which was significantly remedied by treatment with dietary IRW or IQW $(P<0.05)$. Although Fig. 1B indicates that the administration of DSS resulted in considerably more extreme colitis in the colons of mice from the DSS, IRW-DSS, and IQW-DSS groups compared to the control group, the presence of IRW and IQW resulted in significantly reduced $(P<0.05)$ disease scores.

The results of the ABTS assay measuring the plasma antioxidant capacity are presented in Fig. 2. Although the total antioxidant capacity was significantly decreased $(P<0.05)$ in the DSS, IRW-DSS, and IQW-DSS groups relative to the control groups, the total antioxidant capacity in the IRW-DSS and IQW-DSS groups was significantly higher $(P<0.05)$ than that

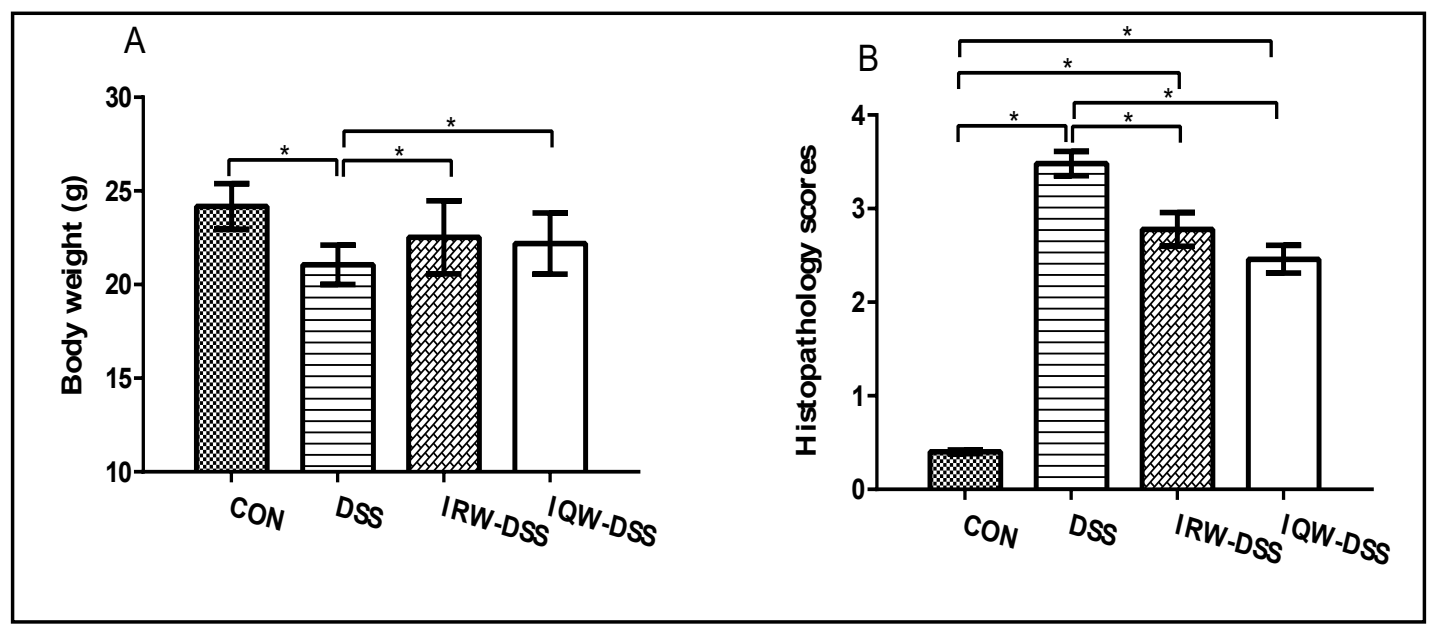

Fig. 1. Influence of dietary IRW and IQW on (A) body weight (g) and (B) colon histopathology scores. The results are expressed as mean \pm SEM. * indicates significant difference between groups $(\mathrm{P}<0.05 ; \mathrm{n}=10)$. 


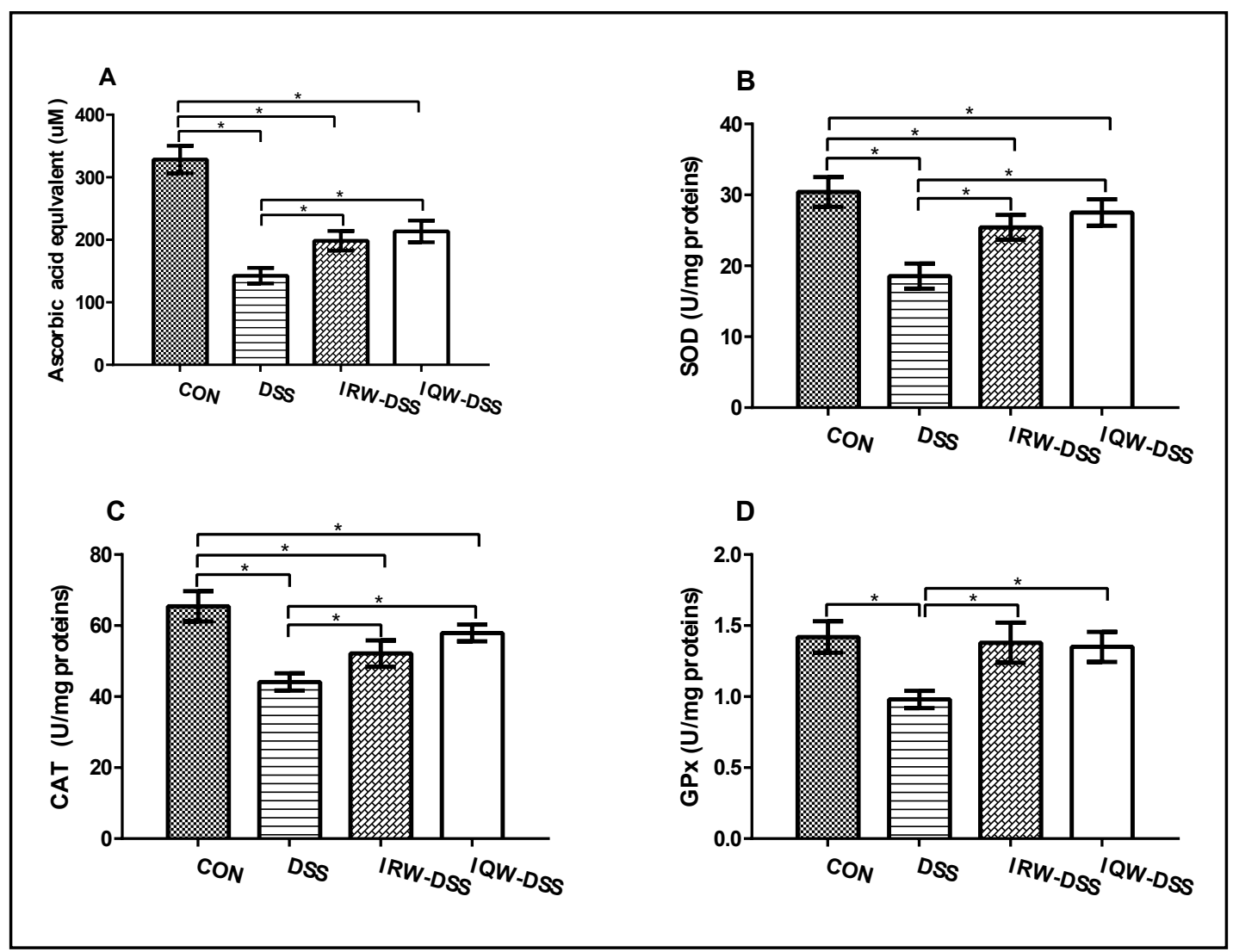

Fig. 2. Antioxidant enzymes in the colons of mice following administration of dietary IRW or IQW. The results are expressed as mean $\pm S E M . *$ indicates significant difference between groups $(\mathrm{P}<0.05 ; n=10)$.

in the DSS group (Fig. 2A). Fig. 2B-D presents the antioxidant enzyme activities. Significant reductions in SOD and CAT activities, along with GPx activity $(P<0.05)$, were indicated for the DSS group relative to the control group. The administration of dietary IRW or IQW led to significant enhancement of SOD, CAT, and GPx activities $(P<0.05)$ relative to the DSS group, although no measurable difference in GPx activities was noted in the control, IRW-DSS, and IQW-DSS groups. These outcomes indicate that the antioxidative effect of dietary MR results in significantly enhanced SOD, CAT, and GPx enzymatic activities.

The V3-V4 regions of the $16 \mathrm{~S}$ rRNA were detected in all of the fecal samples collected at necropsy. In total, 334, 370 eligible sequences were acquired, with samples from the control, DSS, IRW, and IQW groups furnishing 83, 184, 83, 229, 82, 749, and 85, 208 raw tags, respectively. Trimming, assembly, and quality filtering furnished 72, 295, 71, 741, 72, 538, and 74,567 effective tags for analysis, from which $681 \pm 36,631 \pm 50,590 \pm 27$, and $666 \pm 67$ OTUs were respectively detected for the control, DSS, IRW-DSS, and IQW-DSS groups. The selected sequences can adequately determine the great majority of bacterial diversity parameters, based on the normalized subsamples of 51, 568 readings per sample. Table 1 presents the results of the diversity indexes (Shannon and Simpson), community richness (Chao1 and ACE), and coverage (Good's coverage estimator) calculations. Fig. 3A indicates that IRW-DSS treatment significantly enhanced the Shannon index $(P<0.05)$ relative to the control and DSS groups. Fig. 3B indicates that both the IRW-DSS and IQW-DSS significantly enhanced the Simpson index $(P<0.05)$ relative to the control group. The community richness (Chao1 and ACE) and the coverage (Good's coverage estimator) demonstrated no statistically significant differences. 
Table 1. Alpha diversity indices of the fecal bacterial communities of mice. * indicates significant difference between CON vs other groups $(\mathrm{P}<0.05 ; \mathrm{n}=8)$; ${ }^{\#}$ indicate significant difference between DSS vs other groups $(\mathrm{P}<0.05 ; \mathrm{n}=8)$

\begin{tabular}{lcccc}
\hline Item & CON & DSS & IRW & IQW \\
\hline Raw tags & $83,184 \pm 2,041$ & $83,229 \pm 2,407$ & $82,749 \pm 2,569$ & $85,208 \pm 1,613$ \\
Effective tags & $72,295 \pm 1,831$ & $71,741 \pm 2,107$ & $72,538 \pm 2,276$ & $74,567 \pm 1,299$ \\
OTU & $681 \pm 36$ & $631 \pm 50$ & $590 \pm 27$ & $666 \pm 67$ \\
Shannon & $5.46 \pm 0.19$ & $5.54 \pm 0.18$ & $5.87 \pm 0.03$ & $6.23 \pm 0.09^{*}$ \\
Simpson & $0.91 \pm 0.02$ & $0.94 \pm 0.01$ & $0.96 \pm 0.00^{*}$ & $0.96 \pm 0.00^{*}$ \\
Chao1 & $687.57 \pm 21.23$ & $632.29 \pm 46.08$ & $576.50 \pm 7.45$ & $644.88 \pm 63.35$ \\
ACE & $718.55 \pm 20.20$ & $654.22 \pm 49.83$ & $597.58 \pm 3.42$ & $668.48 \pm 68.63$ \\
Coverage & $0.998 \pm 0.000$ & $0.998 \pm 0.000$ & $0.998 \pm 0.000$ & $0.998 \pm 0.000$ \\
\hline
\end{tabular}

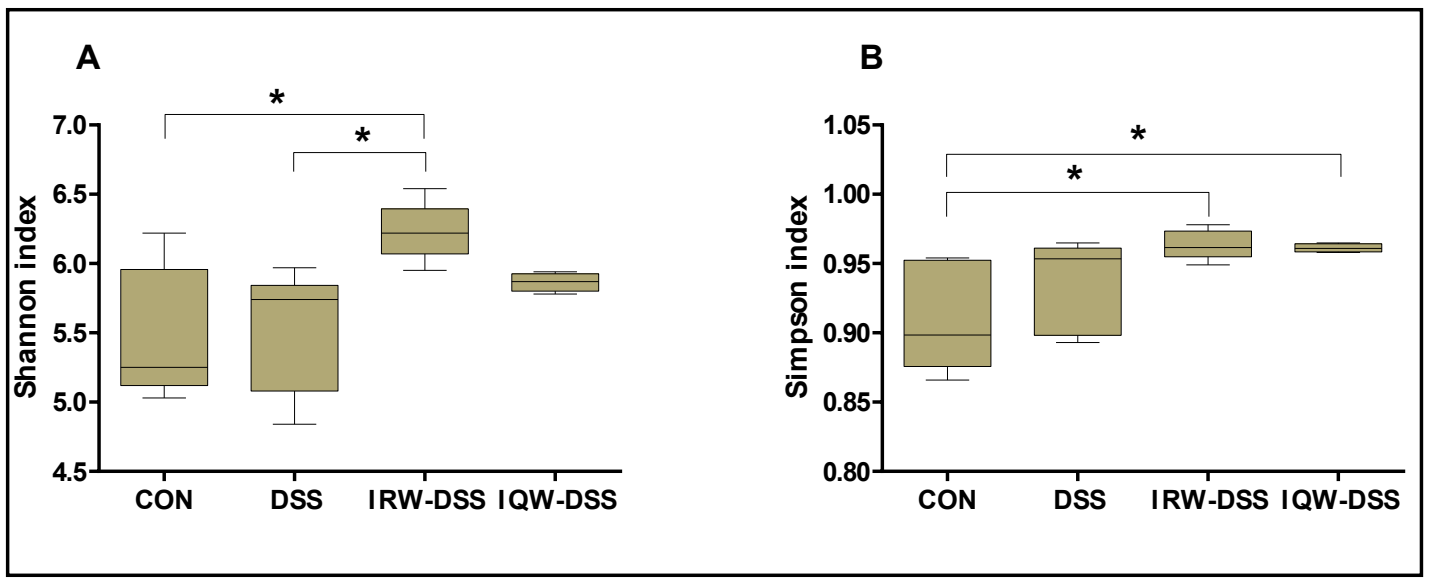

Fig. 3. Shannon and Simpson indexes of colon microbiota in the four different groups. * indicates significant difference between groups $(\mathrm{P}<0.05 ; \mathrm{n}=8)$.

The taxon-dependent analysis was applied to the fecal samples to examine the taxonomy of the intestinal microbiota, leading to the detection of seven phyla. Fig. 4 presents the relative abundance of the detected species, indicating the predominance of Bacteroidetes, Firmicutes, and Proteobacteria. For the control group, the relative abundances were $66.30 \%$ Bacteroidetes, $28.40 \%$ Firmicutes, and 4.21\% Proteobacteria, whereas the DSS group showed $58.93 \%$ Bacteroidetes, 31.46\% Firmicutes, and 7.97\% Proteobacteria. The IRW group revealed relative abundances of $47.31 \%$ Bacteroidetes, $43.76 \%$ Firmicutes, and 7.55\% Proteobacteria. The IQW-DSS group demonstrated relative abundances of $46.24 \%$ Bacteroidetes, $47.07 \%$ Firmicutes, and 5.00\% Proteobacteria.

Table 2 lists the most abundant genera. For the Bacteroidetes phylum, the relative abundance of genus Bacteroides increased in the DSS group relative to the control and IQWDSS groups $(P<0.05)$, whereas the relative abundance of Parabacteroides increased in the IQW group relative to the control and DSS groups $(P<0.05)$. For the Firmicutes phylum, the 
relative abundance of the Coprococcus_1 genus was enhanced by the IQW treatment $(P<0.05)$ relative to the other three groups, whereas the relative abundance of Ruminococcaceae_UCG014 was enhanced by both the IRW and IQW treatments $(P<0.05)$ relative to the control and DSS groups. For the Proteobacteria phylum, the abundance of genus Desulfovibrio was enhanced relative to that of the control group in both the IRW and IQW groups $(P<0.05)$.

Table 2. Composition of the fecal bacterial communities of mice at the genus level. * indicates significant

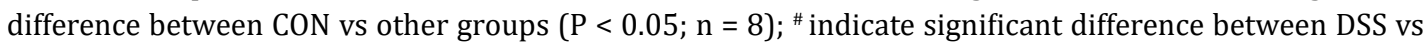
other groups $(\mathrm{P}<0.05 ; \mathrm{n}=8)$

\begin{tabular}{|c|c|c|c|c|c|}
\hline Phyla & Genus & CON & DSS & IRW & IQW \\
\hline \multirow[t]{3}{*}{ Bacteroidetes } & Alloprevotella & $23.86 \pm 4.13^{\#}$ & $11.79 \pm 5.30^{*}$ & $2.16 \pm 0.69^{*}$ & $1.18 \pm 0.24^{*}$ \\
\hline & Bacteroides & $11.46 \pm 1.39 \#$ & $18.49 \pm 2.96^{*}$ & $13.14 \pm 2.03$ & $9.65 \pm 1.50^{\#}$ \\
\hline & Parabacteroides & $2.14 \pm 0.23$ & $2.23 \pm 0.50$ & $1.31 \pm 0.14$ & $1.13 \pm 0.18^{* \#}$ \\
\hline \multirow[t]{9}{*}{ Firmicutes } & Anaerotruncus & $0.36 \pm 0.08$ & $0.51 \pm 0.17$ & $0.83 \pm 0.14^{*}$ & $0.75 \pm 0.11$ \\
\hline & Coprococcus_1 & $0.29 \pm 0.05$ & $0.52 \pm 0.08$ & $0.76 \pm 0.12$ & $1.48 \pm 0.36^{* \#}$ \\
\hline & Erysipelatoclostridium & $1.02 \pm 0.43$ & $1.77 \pm 0.57$ & $0.57 \pm 0.12$ & $0.71 \pm 0.18$ \\
\hline & Lachnoclostridium & $0.88 \pm 0.15$ & $0.92 \pm 0.23$ & $0.89 \pm 0.08$ & $0.76 \pm 0.09$ \\
\hline & Lachnospiraceae_NK4A136_group & $7.07 \pm 1.43$ & $7.82 \pm 2.70$ & $10.01 \pm 2.82$ & $12.24 \pm 3.76$ \\
\hline & Oscillibacter & $0.35 \pm 0.05$ & $0.77 \pm 0.27$ & $1.07 \pm 0.16^{*}$ & $0.83 \pm 0.14$ \\
\hline & Roseburia & $0.70 \pm 0.14$ & $0.78 \pm 0.19$ & $2.43 \pm 1.31$ & $0.74 \pm 0.13$ \\
\hline & Ruminiclostridium_9 & $0.50 \pm 0.01^{\#}$ & $1.55 \pm 0.46^{*}$ & $1.69 \pm 0.27^{*}$ & $1.40 \pm 0.27$ \\
\hline & Ruminococcaceae_UCG-014 & $0.41 \pm 0.07$ & $0.67 \pm 0.05$ & $1.77 \pm 0.16^{* \#}$ & $2.47 \pm 0.49^{* \#}$ \\
\hline \multirow[t]{3}{*}{ Proteobacteria } & Desulfovibrio & $0.38 \pm 0.05$ & $0.70 \pm 0.18$ & $1.08 \pm 0.19^{*}$ & $1.16 \pm 0.28^{*}$ \\
\hline & Helicobacter & $2.05 \pm 0.55$ & $4.80 \pm 1.51$ & $4.47 \pm 0.90$ & $1.53 \pm 0.57 \#$ \\
\hline & Parasutterella & $0.80 \pm 0.23$ & $1.14 \pm 0.39$ & $0.30 \pm 0.05^{\#}$ & $1.24 \pm 0.17$ \\
\hline
\end{tabular}

Fig. 4. Microbiota of the colon in the four groups at the phylum level.

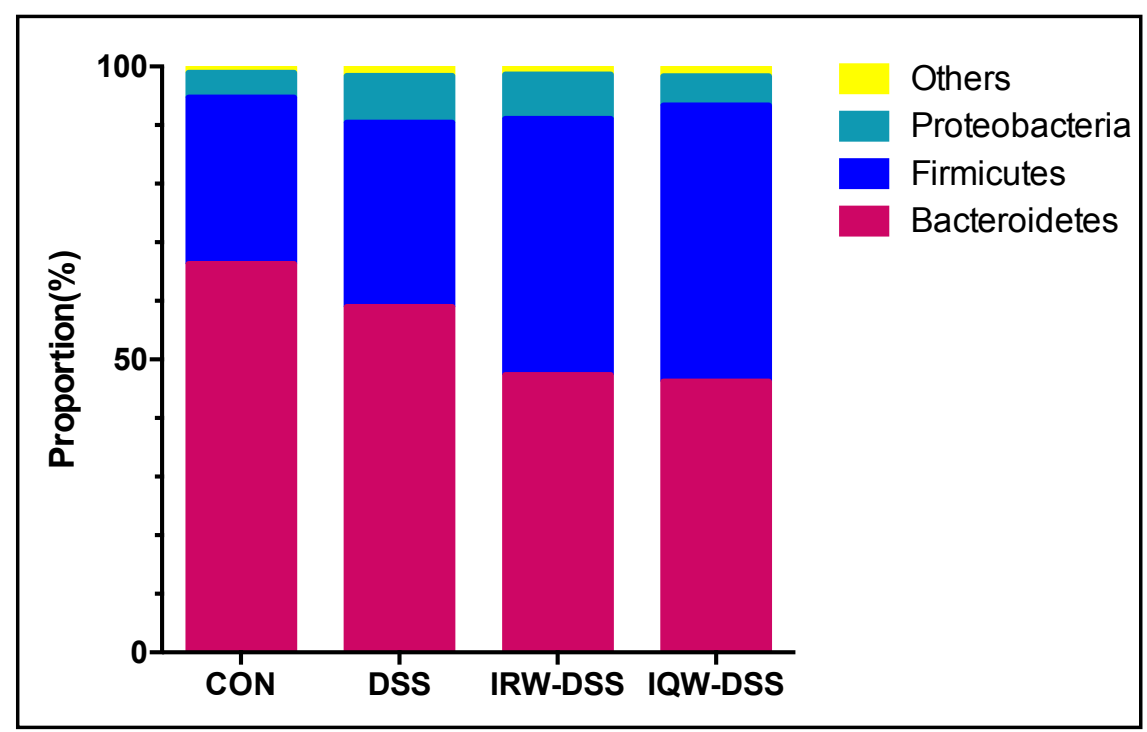




\section{Discussion}

This study examines the impacts of two different peptides (IRW and IQW) on DSSinduced colitis in mice to model the pathology of IBD, a persistent inflammatory condition of the gastrointestinal tract that includes CD and UC. The mice treated with DSS displayed clinical symptoms and pathological developments comparable to those associated with UC in humans, such as body weight reduction, diarrhea, and blood in the feces. The outcomes of this study indicate that IRW and IQW decrease DAI scores, weight loss, and oxidative stress in the presence of DSS, thus significantly inhibiting DSS-induced colitis. Moreover, IRW and IQW increased the diversity of microbial diversity in the colon and decreased the abundance of Bacteroides, but enhanced Parabacteroides, Anaerotruncus, Oscillibacter, and Ruminiclostridium_9.

IBD is linked to significant alterations in the constitution, diversity, and metabolic function of the extensive, diverse, and complex community of microbiota found in the human gut. High-throughput screening was used to assess the microbiological community present in the mice. The diversity of the sample microbiological community, in terms of species richness and species evenness, is indicated by the Shannon and Simpson indices. For communities with identical species richness, an increased diversity is reflected by increased species uniformity. Microbial microecology in the intestine of mice is associated with the development of DSS-induced colitis [21, 25, 26]. Research has found that the compositions and varieties have changed in DSS-induced mouse models, such as those of Bacteroides, Lactobacilli, Parabacteroides, Enterobacteriaceae, and Anaerotruncus [27, 28]. Yeom et al. reported significantly higher Shannon diversity indices for the control group compared to the DSS group; furthermore, DSS increased the ratio of Bacteroidetes, but reduced the proportion of Firmicutes [29]. Ritchie et al. detected no significant differences between the control group and rats treated with DSS at 47 days after treatment and colonic injury showed a negative relation to Lactobacillus, Actinobacteria, Firmicutes, and Lactobacillales [30]. However, at the same amount of time after treatment, DSS rats given Sumac bran displayed significantly enhanced richness $(P=0.0199)$ and measurably greater diversity indices than control rats given Sumac bran. Similarly, DSS rats given Hi Tannin black bran displayed significantly enhanced richness $(P<0.001)$ and diversity $(P=0.013)$ than control rats administered Hi Tannin black bran. These differences may have occurred because the experimental rats were also given an effective experimental diet with a specific function in DSS treatment [30].

Due to the central role of gut microbiota in IBD, sufferers are generally administered short-term antibiotic treatment to ameliorate intestinal inflammation [31, 32]. In the IBD animal model, the proportion of symbiotic organisms, such as Helicobacter, Clostridium, and Enterococcus, has changed [33]. Differences in microbiota among experimental groups were compared at the phylum level by Yeom et al., who reported that the levels of Bacteroidetes were significantly enhanced (by 44.9\%) and the levels of Firmicutes were significantly reduced (by $34.4 \%$ ) relative to the control group following DSS treatment. Accordingly, the Bacteroidetes to Firmicutes ratio in the gut microbiota was greater for the DSS group than for the control group [30,34]. Although the Bacteroidetes and Firmicutes phyla are predominant in healthy gut microbiota, Zhou et al. reported significant reductions in these species, along with significant increases in Proteobacteria and Actinobacteria in patients with IBD [35]. Munyaka et al. detected 14 phyla, 5 of which (Bacteroidetes, Deferribacteres, Firmicutes, Proteobacteria, and Verrucomicrobia) were abundant, constituting more than $1 \%$ of the population. The remaining nine (Acidobacteria, Actinobacteria, Cyanobacteria, Fibrobacteres, Lentisphaerae, Planctomycetes, Spirochaetes, TM7, and Tenericutes) were rare, constituting less than $1 \%$ of the population. No significant differences in the abundant phyla $(P>0.05)$ were noted for the DSS-treated mice and the control group [36]. 


\section{Cellular Physiology Cell Physiol Biochem 2018;51:441-451

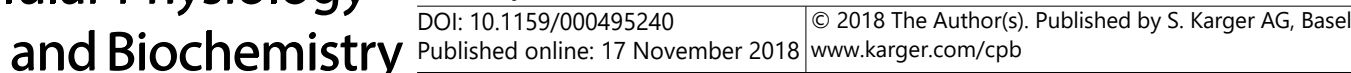 \\ Liu et al.: Effects of IRW and IQW on IBD}

At the genus level, Yeom et al. found that DSS treatment led to significantly enhanced proportions of Clostridium, Bacteroides, and Enterobacter relative to the control group [29]. Kang et al. examined the microbiota in fecal samples, identifying genera that increased in the presence of IBD (Akkermansia, Bacteroides, DQ532165_g, Bifidobacterium, EF406806_g, and Prevotella) and others that decreased under the same condition (DQ326408_f_uc,EF406830_g, EF602759_f_uc, EF602759_g, EF603943_g, EU381732_f_uc, EU381732_g, Erysipelotrichi_uc_g, and Lactobacillus). When the microbiotia communities in the small intestinal fluids were examined at the genus level, the major bacteria (Turibacter, Pseudomonas, and EF406806_g) showed small compositional changes, their respective abundances on the first day being $54.2 \%, 16.5 \%$, and $6.1 \%$ and on the second day being $54.1 \%, 15.3 \%$, and $8.1 \%$, respectively. By contrast, the abundance of EF602759_f_uc decreased in the presence of IBD from $9.2 \%$ on day 1 to $0.7 \%$ on day 2 [37]. The differences in the results of Kang et al. and those of this study may be due to the former's use of the metagenomic analysis method and the fact that they examined samples from small intestinal fluids. Moreover, the up-regulation of antimicrobial peptides can mediate the composition of intestinal microorganisms and protect the host from foodborne and other pathogens in the IBD model $[38,39]$.

\section{Conclusion}

In brief, this study indicates that 7 days of treatment with DSS induces symptoms of colitis, such as reduced growth and antioxidant enzyme inhibition, whereas the provision of dietary IRW or IQW ameliorates these effects by up-regulating the antioxidant enzymes, thus relieving oxidative stress. Hence, this study concludes that dietary IRW or IQW may provide a subsidiary treatment for IBD patients. Changes in alpha-diversity in IBD following treatment with DSS, IRW, and IQW, along with species changes at the phylum and genus levels, are also examined. Although the impacts of dietary IRW and IQW on DSS-induced colitis and the microbiota of the gut are demonstrated, the relationship between the antioxidant effects of IRW and IQW and the microbiota of the gut remains unresolved.

\section{Acknowledgements}

This study was supported by the National Natural Science Foundation of China (Nos. 31672457 and 31772642), the National Key Research and Development Program of China (No. 2016YFD0500504 and 2018YFD0501003), Local Science and Technology Development Project Guided by The Central Government (YDZX20184300002303, 2018CT5002), the Ministry of Agriculture of the People's Republic of China (Nos. 2015-Z64 and 2016-X47), the Hunan Provincial Science and Technology Department (Nos. 2016NK2101, 2016WK2008, 2016TP2005, and 2017NK2322), and China Postdoctoral Science Foundation 2018M632963.

\section{Disclosure Statement}

All co-authors are aware of and agree with the contents of this manuscript. There is no financial interest to report. 


\section{Cellular Physiology Cell Physiol Biochem 2018;51:441-451 \begin{tabular}{l|l|l|l|l}
\hline DOI: 10.1159/000495240 2018 The Author(s). Published by S. Karger AG, Basel & (c) Biochemistry
\end{tabular}

\section{References}

1 Michalčíková RB, Dryahina K, Španěl P: SIFT-MS quantification of several breath biomarkers of inflammatory bowel disease, IBD: A detailed study of the ion chemistry. International Journal of Mass Spectrometry 2016;396:35-41.

- Kronman MP, Gerber JS, Prasad PA, Adler AL, Bass JA, Newland JG, Shah KM, Zerr DM, Feng R, Coffin SE: Variation in Antibiotic Use for Children Hospitalized With Inflammatory Bowel Disease Exacerbation: A Multicenter Validation Study. Journal of the Pediatric Infectious Diseases Society 2012;1:306.

-3 Bager P, Simonsen J, Nielsen NM, Frisch M: Cesarean section and offspring's risk of inflammatory bowel disease: a national cohort study. Inflammatory Bowel Diseases 2012;18:857-862.

4 Manichanh C, Borruel N, Casellas F, Guarner F: The gut microbiota in IBD. Nature Reviews Gastroenterology \& Hepatology 2012;9:599.

75 Kostic AD, Xavier RJ, Gevers D: The Microbiome in Inflammatory Bowel Diseases: Current Status and the Future Ahead. Gastroenterology 2014;146:1489-1499.

6 Nagalingam NA, Lynch SV: Role of the microbiota in inflammatory bowel diseases. Inflammatory Bowel Diseases 2012;18:968-984.

7 Pender SL: Do metalloproteinases contribute to tissue destruction or remodeling in the inflamed gut? Inflammatory Bowel Diseases 2010;14:S136-S137.

8 Goyal N, Shukla G: Probiotic Lactobacillus rhamnosus GG Modulates the Mucosal Immune Response in Giardia intestinalis- Infected BALB/c Mice. Digestive Diseases \& Sciences 2013;58:1218-1225.

-9 Llopis M, Antolin M, Carol M, Borruel N, Casellas F, Martinez C, Espín-Basany E, Guarner F, Malagelada JR: Lactobacillus casei downregulates commensals' inflammatory signals in Crohn's disease mucosa. Inflammatory Bowel Diseases 2009;15:275-283.

10 Martín R, Miquel S, Chain F, Natividad JM, Jury J, Lu J, Sokol H, Theodorou V, Bercik P, Verdu EF: Faecalibacterium prausnitzii prevents physiological damages in a chronic low-grade inflammation murine model. Bmc Microbiology 2015;15:67.

-11 Nishiyama Y, Kataoka T, Yamato K, Taguchi T, Yamaoka K: Suppression of dextran sulfate sodium-induced colitis in mice by radon inhalation. Mediators Inflamm 2012;2012:239617.

12 McLean LP, Cross RK: Adverse events in IBD: to stop or continue immune suppressant and biologic treatment. Expert Rev Gastroenterol Hepatol 2014;8:223-240.

13 Majumder K, Wu JP: A new approach for identification of novel antihypertensive peptides from egg proteins by QSAR and bioinformatics. Food Research International 2010;43:1371-1378.

14 Chen S, Jiang HM, Peng HH, Wu XS, Fang J: The Utility of Ovotransferrin and Ovotransferrin-Derived Peptides as Possible Candidates in the Clinical Treatment of Cardiovascular Diseases. Oxidative Medicine and Cellular Longevity 2017;Artn 6504518.10.1155/2017/6504518

15 Kaustav M, Subhadeep C, Davidge ST, Wu J: Structure and Activity Study of Egg Protein Ovotransferrin Derived Peptides (IRW and IQW) on Endothelial Inflammatory Response and Oxidative Stress. Journal of Agricultural \& Food Chemistry 2013;61:2120-2129.

16 Huang W, Shen S, Nimalaratne C, Li S, Majumder K, Wu J: Effects of addition of egg ovotransferrin-derived peptides on the oxygen radical absorbance capacity of different teas. Food Chemistry 2012;135:1600-1607.

17 Ren W, Yin J, Wu M, Liu G, Yang G, Xion Y, Su D, Wu L, Li T, Chen S, Duan J, Yin Y, Wu G: Serum amino acids profile and the beneficial effects of L-arginine or L-glutamine supplementation in dextran sulfate sodium colitis. PLoS One 2014;9:e88335.

18 He F, Wu C, Li P, Li N, Zhang D, Zhu Q, Ren W, Peng Y: Functions and Signaling Pathways of Amino Acids in Intestinal Inflammation. Biomed Res Int 2018;2018:9171905.

-19 Zhou X, Zhang Y, Wu X, Wan D, Yin Y: Effects of Dietary Serine Supplementation on Intestinal Integrity, Inflammation and Oxidative Status in Early-Weaned Piglets. Cell Physiol Biochem 2018;48:993-1002.

20 Re R, Pellegrini N, Proteggente A, Pannala A, Yang M, Rice-Evans C: Antioxidant activity applying an improved ABTS radical cation decolorization assay. Free Radical Biology and Medicine 1999;26:1231-1237.

-21 Yin J, Han H, Li Y, Liu Z, Zhao Y, Fang R, Huang X, Zheng J, Ren W, Wu F, Liu G, Wu X, Wang K, Sun L, Li C, Li T, Yin Y: Lysine Restriction Affects Feed Intake and Amino Acid Metabolism via Gut Microbiome in Piglets. Cell Physiol Biochem 2017;44:1749-1761. 


\section{Cellular Physiology Cell Physiol Biochem 2018;51:441-451

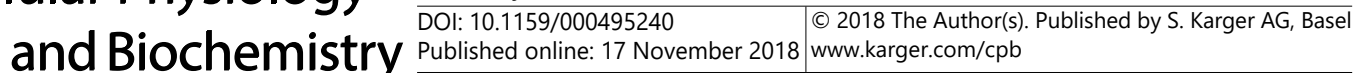

Liu et al.: Effects of IRW and IQW on IBD

-22 Caporaso JG, Kuczynski J, Stombaugh J, Bittinger K, Bushman FD, Costello EK, Fierer N, Pena AG, Goodrich JK, Gordon JI, Huttley GA, Kelley ST, Knights D, Koenig JE, Ley RE, Lozupone CA, McDonald D, Muegge BD, Pirrung M, Reeder J et al.: QIIME allows analysis of high-throughput community sequencing data. Nat Methods 2010;7:335-336.

23 Magoc T, Salzberg SL: FLASH: fast length adjustment of short reads to improve genome assemblies. Bioinformatics 2011;27:2957-2963.

-24 Wang Q, Garrity GM, Tiedje JM, Cole JR: Naive Bayesian classifier for rapid assignment of rRNA sequences into the new bacterial taxonomy. Appl Environ Microbiol 2007;73:5261-5267.

-25 Nagalingam NA, Kao JY, Young VB: Microbial ecology of the murine gut associated with the development of dextran sodium sulfate-induced colitis. Inflamm Bowel Dis 2011;17:917-926.

-26 Zhu D, Ma Y, Ding S, Jiang H, Fang J: Effects of Melatonin on Intestinal Microbiota and Oxidative Stress in Colitis Mice. Biomed Res Int 2018;2018:2607679.

-27 Hakansson A, Tormo-Badia N, Baridi A, Xu J, Molin G, Hagslatt ML, Karlsson C, Jeppsson B, Cilio CM, Ahrne S: Immunological alteration and changes of gut microbiota after dextran sulfate sodium (DSS) administration in mice. Clin Exp Med 2015;15:107-120.

-28 Munyaka PM, Rabbi MF, Khafipour E, Ghia JE: Acute dextran sulfate sodium (DSS)-induced colitis promotes gut microbial dysbiosis in mice. J Basic Microbiol 2016;56:986-998.

29 Yeom Y, Kim BS, Kim SJ, Kim Y: Sasa quelpaertensis leaf extract regulates microbial dysbiosis by modulating the composition and diversity of the microbiota in dextran sulfate sodium-induced colitis mice. BMC Complement Altern Med 2016;16:481.

-30 Ritchie LE, Sturino JM, Carroll RJ, Rooney LW, Azcarate-Peril MA, Turner ND: Polyphenol-rich sorghum brans alter colon microbiota and impact species diversity and species richness after multiple bouts of dextran sodium sulfate-induced colitis. FEMS Microbiol Ecol 2015;91:fiv008.

-31 Collins SM, Bercik P: The relationship between intestinal microbiota and the central nervous system in normal gastrointestinal function and disease. Gastroenterology 2009;136:2003-2014.

32 Triantafillidis JK, Merikas E, Georgopoulos F: Current and emerging drugs for the treatment of inflammatory bowel disease. Drug design, development and therapy 2011;5:185.

33 Round JL, Mazmanian SK: The gut microbiota shapes intestinal immune responses during health and disease. Nature Reviews Immunology 2009;9:313.

-34 Sokol H, Seksik P, Furet J, Firmesse O, Nion - Larmurier I, Beaugerie L, Cosnes J, Corthier G, Marteau P, Doré J: Low counts of Faecalibacterium prausnitzii in colitis microbiota. Inflammatory bowel diseases 2009;15:1183-1189.

-35 Zhou MX, He J, Shen YJ, Zhang C, Wang JZ, Chen YW: New Frontiers in Genetics, Gut Microbiota, and Immunity: A Rosetta Stone for the Pathogenesis of Inflammatory Bowel Disease. Biomed Research International 2017;Artn 8201672. doi: 10.1155/2017/8201672.

-36 Munyaka PM, Rabbi MF, Khafipour E, Ghia JE: Acute dextran sulfate sodium (DSS)-induced colitis promotes gut microbial dysbiosis in mice. J Basic Microbiol 2016;56:986-998.

-37 Kang CS, Ban M, Choi EJ, Moon HG, Jeon JS, Kim DK, Park SK, Jeon SG, Roh TY, Myung SJ, Gho YS, Kim JG, Kim YK: Extracellular Vesicles Derived from Gut Microbiota, Especially Akkermansia muciniphila, Protect the Progression of Dextran Sulfate Sodium-Induced Colitis. Plos One 2013;8:e76520.

-38 Png CW, Lindén SK, Gilshenan KS, Zoetendal EG, McSweeney CS, Sly LI, McGuckin MA, Florin TH: Mucolytic bacteria with increased prevalence in IBD mucosa augment in vitro utilization of mucin by other bacteria. The American journal of gastroenterology 2010;105:2420.

39 Wehkamp J, Stange EF: Is there a role for defensins in IBD? Inflammatory bowel diseases 2008;14:S85-S87. 